\title{
Gestational hyperglycemia reprograms cardiac gene expression in rat offspring
}

\author{
Lara Lehtoranta ${ }^{1}$, Anna Koskinen ${ }^{1}$, Olli Vuolteenaho ${ }^{1}$, Jukka Laine ${ }^{1}$, Ville Kytö ${ }^{1}$, Hanna Soukka ${ }^{1}$, Eeva Ekholm ${ }^{1}$ and \\ Juha Räsänen ${ }^{1}$
}

BACKGROUND: Rat fetuses with maternal pregestational hyperglycemia develop cardiac dysfunction, and their cardiac gene expression differs from that of healthy control fetuses near term. We hypothesized that cardiac gene expression and morphologic abnormalities of rat fetuses with maternal pregestational hyperglycemia become normal after birth.

METHODS: Nine rats were preconceptually injected with streptozotocin to induce maternal hyperglycemia and nine rats served as controls. The hyperglycemia group comprised 82 mice and the control group 74 offspring fed by euglycemic dams. Hearts of the offspring were collected on postnatal days 0,7 , and 14, and processed for histologic and gene expression analyses.

RESULTS: On day 0, heart weight was increased, and expression of cardiac genes involved in contractility, growth, and metabolism was decreased in the hyperglycemia group. On day 7, although cardiomyocyte apoptosis was enhanced, most of the changes in gene expression had normalized in the hyperglycemia group. By day 14, the expression of genes important for myocardial growth, function, and metabolism was again abnormal in the hyperglycemia group.

CONCLUSION: Most cardiac gene expression abnormalities become transiently normal during the first week of life of offspring to hyperglycemic rats. However, by day 14, cardiac expressions of genes involved in growth, function, and metabolism are again abnormal in relation to control offspring.

$\mathbf{F}$ etal heart grows mainly through proliferation that is induced by an increase in arterial blood pressure, together with elevated circulating insulin-like growth factor 1 and natriuretic peptide concentrations (1). After birth, the cardiac growth pattern gradually shifts from hyperplastic to hypertrophic. In rats, this shift occurs on postnatal days (PND) 3 and 4 (ref. 2).

In human diabetic pregnancies, optimal maternal glycemic control does not prevent fetal interventricular septal thickening (3). An increased cardiac wall thickness is observed in $25-75 \%$ of newborns of diabetic mothers $(4,5)$. The severity of this cardiomyopathy can vary from an incidental finding on echocardiography to serious symptoms of congestive heart failure (6). Usually, however, the increased cardiac mass is transient and resolves within a few months $(4,5,7)$. In a mouse model of maternal pregestational hyperglycemia (HG), fetal septal and left ventricular wall thicknesses were transiently increased near term gestation, and cardiac growth became normal by the age of 1 week (ref. 8).

We have shown in a rat model of maternal pregestational HG that fetal cardiac dysfunction is present during the second half of pregnancy and that cardiac gene expression patterns deviate from normal fetuses near term (9). On the basis of these observations, we hypothesized that cardiac gene expression and the morphologic abnormalities in fetuses of rats with pregestational HG will become normal during the neonatal period.

\section{METHODS}

\section{Animals, Induction of HG, and Data Acquisition}

Animal care followed the guidelines set by the European Community Council Directions 86/609/EC. The study protocol was approved by the Turku University Ethics Committee for animal experiments and the University of Turku Laboratory Animal Care and Use Committee (permission no. 1479/2005 of the Animal Experiment Board of Finland). The rats were purchased from the University of Turku Central Animal Laboratory and housed in specific pathogen-free conditions in room air with a 14:10 h light-dark cycle, and they had free access to food and water.

HG was induced with an intraperitoneal injection of $35 \mathrm{mg} / \mathrm{kg}$ of streptozotocin (Sigma, St Louis, MO) dissolved in citrate buffer $(0.01 \mathrm{~mol} / \mathrm{l}, \mathrm{pH} 4.5)$ in 9 female Sprague-Dawley rats prior to mating (10). Nine untreated female Sprague-Dawley rats served as euglycemic controls. Female rats were caged overnight with a male. The day a positive vaginal smear was obtained was designated as gestational day 1. Streptozotocin-treated rats had consistent HG before $(20.9 \pm 2.1 \mathrm{mmol} / \mathrm{l})$ and during pregnancy $(25.0 \pm 3.8 \mathrm{mmol} /$ $1)$, whereas control dams were euglycemic before $(5.1 \pm 0.2 \mathrm{mmol})$ and during gestation $(4.0 \pm 0.5 \mathrm{mmol} / \mathrm{l})$. The dams delivered spontaneously at term (22 days after conception). Newborns were instantly separated from their own dams and fed by healthy euglycemic dams (mean blood glucose $5.4 \pm 2.1 \mathrm{mmol} / \mathrm{l}$ ). Glucose samples were collected from the tail vein of conscious animals and analyzed using an Elite glucometer (Bayer, Leverkusen, Germany). The number of offspring in this study was 82 in the HG and 74 in the control group. 


\section{Maternal hyperglycemia and offspring heart Articles}

Table 1. Neonatal heart and body weights

\begin{tabular}{|c|c|c|c|c|c|c|}
\hline \multirow[t]{2}{*}{ Variable } & \multicolumn{3}{|c|}{ Control } & \multicolumn{3}{|c|}{ Hyperglycemia } \\
\hline & PND0 & PND7 & PND14 & PNDO & PND7 & PND14 \\
\hline Number of pups & 20 & 28 & 27 & 28 & 24 & 21 \\
\hline Number of dams & 2 & 3 & 4 & 3 & 3 & 4 \\
\hline Heart weight (mg) & $0.030 \pm 0.005$ & $0.082 \pm 0.010$ & $0.15 \pm 0.01$ & $0.039 \pm 0.004^{* *}$ & $0.076 \pm 0.009$ & $0.15 \pm 0.01$ \\
\hline Body weight (mg) & $5.8 \pm 0.3$ & $16.1 \pm 2.5$ & $30.8 \pm 4.7$ & $5.8 \pm 1.0$ & $12.1 \pm 1.6^{\dagger}$ & $23.4 \pm 4.3^{\dagger}$ \\
\hline Heart/body weight (mg) & $0.0053 \pm 0.0008$ & $0.0057 \pm 0.0007$ & $0.0055 \pm 0.0004$ & $0.0064 \pm 0.0009^{*}$ & $0.0060 \pm 0.0006$ & $0.0056 \pm 0.0005$ \\
\hline
\end{tabular}

PND, postnatal day.

Data are given as means \pm s.d.

${ }^{*} P<0.05 ;{ }^{* *} P<0.001,{ }^{\dagger} P<0.0001$.

The offspring were killed either at PND0 (day of birth), PND7, or PND14. They were anesthetized with a rapid intraperitoneal injection of pentobarbital sodium $(200 \mathrm{mg} / \mathrm{kg}$, Nembutal; Algin BV, Maassluis, the Netherlands) and killed by cervical dislocation. PND14 was selected as the maximum length of postnatal follow-up because lung alveolarization is completed by that age, which suggests full maturation of the cardiorespiratory system (11). Hearts and bodies were weighted and randomly processed for analyses. The trachea was ligated, and the heart and lung blocks were removed under continuous distending pressure. The heart and lung blocks were either processed for histological studies or frozen rapidly in liquid nitrogen and stored at $-80^{\circ} \mathrm{C}$.

\section{Histology}

Heart samples were fixed for $24 \mathrm{~h}$ in $10 \%$ buffered formalin and embedded in paraffin. All samples were cut in $5 \mu \mathrm{m}$ sections, stained, and examined under UV light. The grid was set in the septum near the apex of the heart (excluding the epicardium and the endocardium), and morphology of the cardiomyocytes (12) was examined. A total of five adjacent areas in two sections per heart were examined by a pathologist blinded to the study group (J.L.). The regions investigated were comparable between the groups. Mitotic cells were identified in hematoxylin-eosin-stained samples by their dividing chromatin figures (metaphase and anaphase most clearly identified) and counted (number of mitotic cells $/ \mathrm{mm}^{2}$ ). Apoptotic cells were identified by their dense, hyperchromatic, and fragmented nuclei, eosinophilic cytoplasm, and a clear identifying halo (13). The apoptotic myocardial cells were assessed by the TUNEL assay by a single investigator blinded to the experimental group (V.K.) (14). Briefly, apoptotic nuclear DNA strand breaks were end-labeled with digoxigenin-conjugated dideoxyUTP by terminal transferase, and digoxigenin antibody conjugated to alkaline phosphatase was used to immunohistochemically visualize the breaks. Adjacent tissue sections treated with DNase I to induce DNA fragmentation served as a positive control for apoptosis. The proportion of TUNEL-positive cardiomyocytes was calculated from transverse left ventricular tissue sections using light microscopy $(\times 200$ magnification) with an ocular grid. Apoptotic cardiomyocyte cells were identified by myofilaments surrounding the nucleus. Silver staining was used to count the number of cardiomyocyte nuclei per $\mathrm{mm}^{2}$ and to measure cardiomyocyte width from the narrowest nucleus-crossing dimension. This was done by a single investigator blinded to the experimental group (J.L.) (15). To further investigate the cell turnover rates, we calculated the mitosis-karyorrhexis index from hematoxylin-eosin samples by adding the number of mitotic and apoptotic cells together (16).

Quantitative Reverse Transcription-Polymerase Chain Reaction Total RNA was purified from HG and control offspring whole-heart samples using Qiagen RNeasy reagents (Qiagen, Hilden, Germany). There were 11 control and $14 \mathrm{HG}$ samples at PND0, 13 and 15 at PND7, and 14 and 13 at PND14. First-strand cDNA was synthesized from RNA using Moloney murine leukemia virus reverse transcriptase obtained from Promega, Madison, WI. The PCR reactions were performed with an ABI 7300 Real-Time PCR System using TaqMan chemistry (Applied Biosystems, Foster City, CA). The standard curve method was used for quantification, and the results were normalized to 18S RNA measured from the same samples (17). The primers and bifunctional fluorogenic probes and identification numbers listed in Supplementary Table S1 online (6-carboxyfluorescein and tetramethylrhodamine) were designed with the Primer Express software (Applied Biosystems). The markers were selected according to their function and association with the fetal gene expression program (18) and our previous results in rat fetal hearts (9).

\section{Statistical Analyses}

All data were analyzed using one-way analysis of variance, with the dam as a random effect, or using Student's $t$-test or the MannWhitney $U$-test when appropriate. Analyses tested the significances of the mean differences in the outcome variables. Repeated measurements of gene expression and histologic data were analyzed using time, maternal $\mathrm{HG}$, and interaction between the two as independent variables. The statistical analyses were performed using SAS (version 9.2; SAS Institute, Cary, NC). Statistical significance was set at $P \leq 0.05$. The results are presented as mean \pm s.d..

\section{RESULTS}

\section{Hearts on the Day of Delivery}

At birth, heart and body weights and the heart-to-body weight ratio were increased in the maternal pregestational HG group compared with those in controls (Table 1). However, there were no differences in cell turnover parameters or in cell width measurements and in the number of cell nuclei between the groups (Table 2 and Figure 1).

Concerning genes related to cardiac growth, the expression of the tumor necrosis factor receptor superfamily member $12 \mathrm{a}$ (Tnfrsf12a), which is involved in cardiomyocyte proliferation, was decreased in the HG group, whereas hypoxia inducible factor (HIF)-prolyl hydroxylase $3(E g \ln 3)$, involved in apoptosis, was similarly expressed in both groups. The expression of myocardial adult skeletal myosin heavy chain 2 (Myh2) and $\alpha$-cardiac myosin heavy chain 6 (Myh6) was decreased in the HG group, whereas the expression of embryonic skeletal myosin heavy chain 3 (Myh3) did not differ between the groups. In the HG group, the expression of the natriuretic peptide gene type b $(N p p b)$ was increased, whereas the expression of the atrial natriuretic peptide gene (Nppa) was similar in both groups (Figure 2). In the HG group, the expression of sarcoplasmic reticulum $\mathrm{Ca}^{2+}$-ATPase (Atp2a2, or Serca2a), which controls cardiomyocyte relaxation and contraction, was decreased as was the 


\section{Articles | Lehtoranta et al.}

Table 2. Neonatal cardiomyocyte cell cycle

\begin{tabular}{|c|c|c|c|c|c|c|}
\hline \multirow[t]{2}{*}{ Variable } & \multicolumn{3}{|c|}{ Control } & \multicolumn{3}{|c|}{ Hyperglycemia } \\
\hline & PND0 & PND7 & PND14 & PND0 & PND7 & PND14 \\
\hline Number of pups & 11 & 15 & 10 & 14 & 12 & 14 \\
\hline Number of dams & 2 & 3 & 3 & 3 & 2 & 3 \\
\hline \multicolumn{7}{|l|}{$H \& E$} \\
\hline Mitotic cells (no. of nuclei/mm²) & $5.36 \pm 4.10$ & $6.17 \pm 3.41$ & $1.50 \pm 0.97$ & $5.44 \pm 3.16$ & $6.58 \pm 2.94$ & $2.86 \pm 1.85^{*}$ \\
\hline Apoptotic cells (no. of nuclei/mm²) & $1.27 \pm 1.17$ & $0.57 \pm 0.56$ & $0.30 \pm 0.26$ & $0.82 \pm 0.75$ & $0.67 \pm 0.62$ & $0.64 \pm 0.66$ \\
\hline MKI & $6.64 \pm 4.92$ & $6.73 \pm 3.65$ & $1.80 \pm 1.14$ & $6.26 \pm 3.55$ & $7.25 \pm 3.14$ & $3.50 \pm 1.75^{*}$ \\
\hline \multicolumn{7}{|l|}{ Gömöri } \\
\hline Cell size $(\mu \mathrm{m})$ & $7.06 \pm 1.10$ & $7.36 \pm 0.50$ & $8.44 \pm 1.26$ & $6.50 \pm 0.64$ & $6.96 \pm 0.55$ & $7.84 \pm 0.89$ \\
\hline Nuclear count (no. of nuclei $/ \mathrm{mm}^{2}$ ) & $5900 \pm 733$ & $5321 \pm 759$ & $5357 \pm 1370$ & $6025 \pm 910$ & $5691 \pm 554$ & $4818 \pm 794$ \\
\hline
\end{tabular}

TUNEL

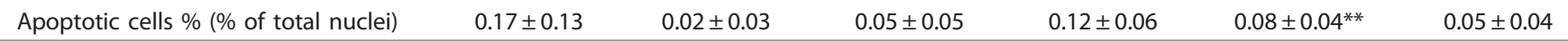

H\&E, hematoxylin and eosin; MKI, mitosis-karyorrhexis index; PND, postnatal day; TUNEL, terminal deoxynucleotidyl transferase nick-end labeling.

Data are given as means \pm s.d.

${ }^{*} P<0.05 ;{ }^{* *} P<0.001$.

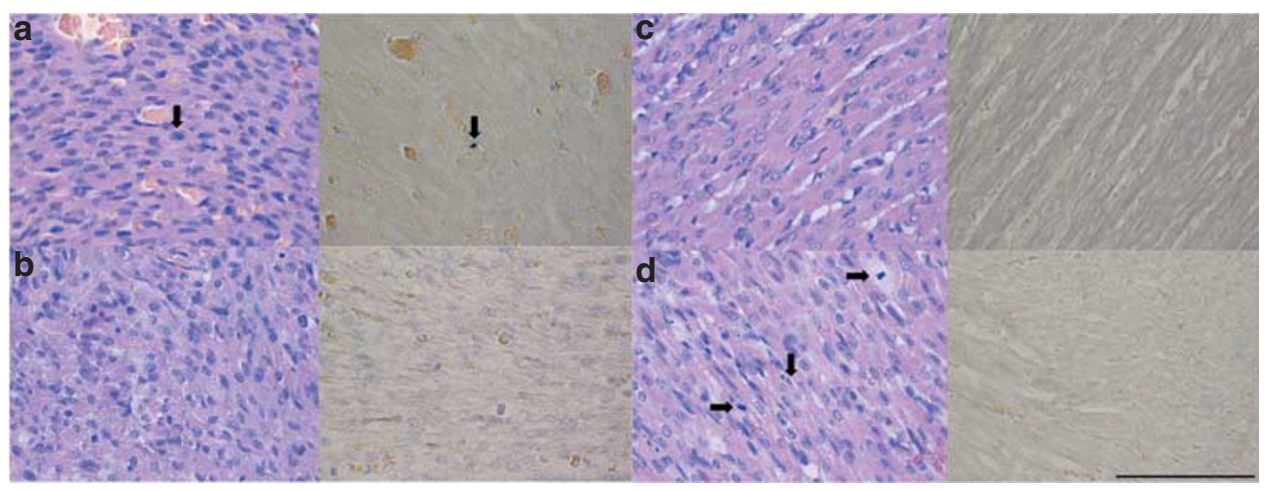

Figure 1. Histology of rat offspring myocardium at PNDO and 14. On the left is a hematoxylin-eosin (H\&E)-stained sample and on the right a TUNEL sample. Scale bar on bottom right $=100 \mu \mathrm{m}$. (a) Control myocardium of newborn (PND0). An apoptotic cell is seen (downward arrow). (b) Myocardium from a newborn of hyperglycemia (HG) dam (PND0). There are no apoptotic cells, but myocardial cells are notably disorganized. (c) Myocardium of control offspring at PND14. No mitotic or apoptotic cells are seen, the cells are clearly aligned, and striation is clearly seen. (d) Myocardium of offspring of a HG dam at PND14. Mitotic cells (rightward arrow) and an apoptotic cell (downward arrow) are seen. Striation is less well defined than that in control offspring at PND14 (frame C). PND, postnatal day; TUNEL, terminal deoxynucleotidyl transferase nick-end labeling.

expression of $\mathrm{Kv}$ channel-interacting protein 2 (Kcnip2), which is important for controlling cardiac electrical function. Maternal HG affected the expression of genes related to cardiac metabolism. The expression of the facilitated (Slc2a3) and the insulin-responsive glucose transporters (Slc2a4) and of uncoupling protein 3 (Ucp3) was decreased in the HG group, whereas the expression of Ucp2 was similar between the groups.

\section{Neonatal Hearts 1 Week after Delivery}

At the age of 1 week, the neonatal rat heart weights of the HG group were similar to those in the control groups, but the body weight was lower in the HG group (Table 1). Again, at this time there were no differences in cell size and turnover parameters between the groups; however, the number of apoptotic cells was higher in the HG group compared with controls (Table 2).

Most of the changes in cardiac gene expression at birth had become normal in the HG group by week 1 after delivery (Figure 2). The expression of Slc2a4 was still lower in the HG group, and, interestingly, the expression of Myh3 had decreased in the HG group relative to the control group.

\section{Offspring Hearts 2 Weeks after Delivery}

At the age of 2 weeks, the heart and body weights and the heart-to-body weight ratio were similar in both groups 


\section{Maternal hyperglycemia and offspring heart | Articles}
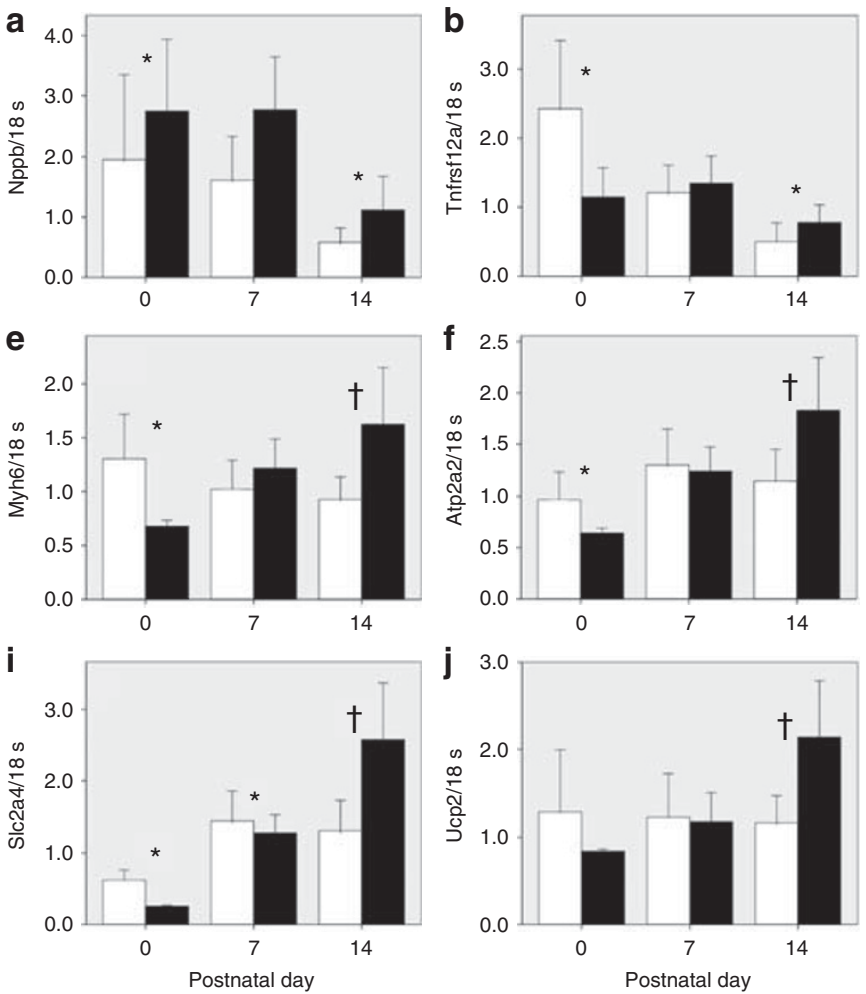
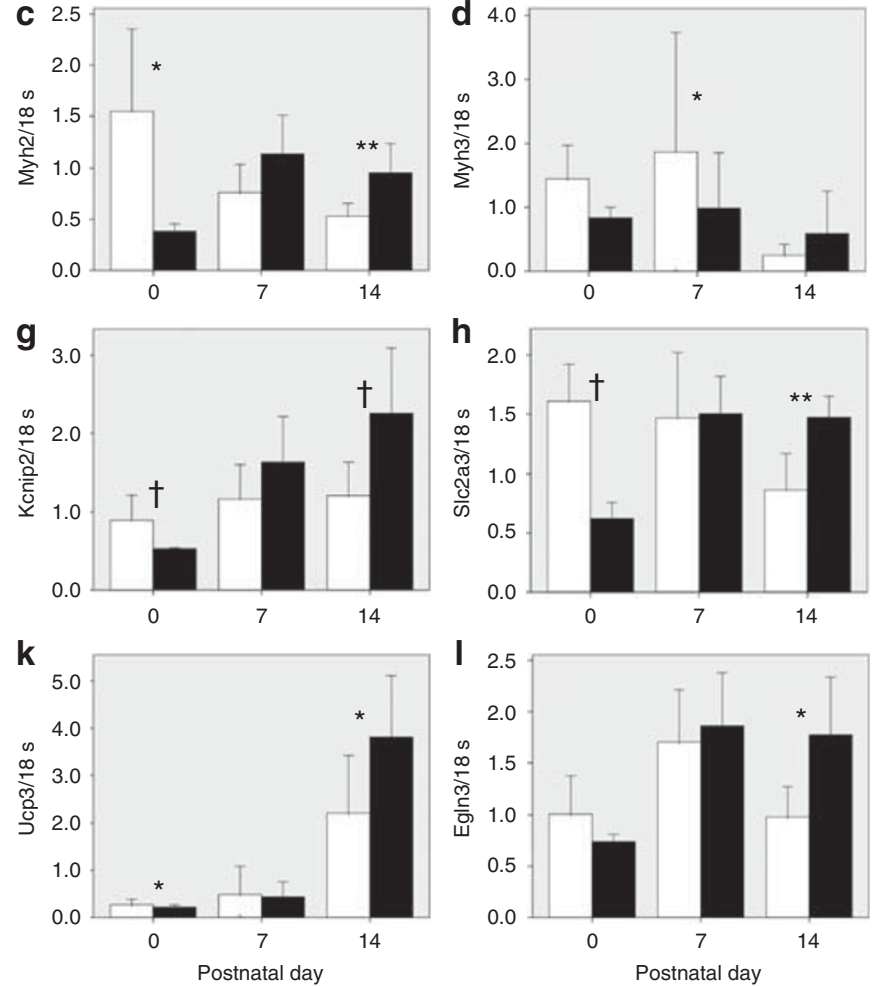

Figure 2. Cardiac gene expression at postnatal days 0,7 , and 14 (gene/18S). White columns represent the control group and black columns the hyperglycemia (HG) offspring gene expression. (a) Nppb, B-type natriuretic peptide gene; (b) Tnfrsf12a, tumor necrosis factor receptor superfamily, member 12a; (c) Myh2, adult skeletal heavy chain myosin-2; (d) Myh3, embryonic skeletal heavy chain myosin-3; (e) Myh6, alpha cardiac heavy chain myosin-6; (f) Atp2a2, sarcoplasmic reticulum Ca2+-ATPase; (g) Kcnip2, Kv channel-interacting protein 2; (h) Slc2a3, facilitated glucose transporter 3; (i) Slc2a4, insulin-responsive glucose transporter 4; (j) Ucp2, uncoupling protein 2; (k) Ucp3, uncoupling protein 3; (I) Egln3, HIF-prolyl hydroxylase 3/egl nine homolog 3. mRNA concentrations are given as arbitrary units (AU). Data are presented as mean $\pm \mathrm{s} . \mathrm{d}$. ${ }^{*} P \leq 0.05 ;{ }^{* *} P<0.001,{ }^{\dagger} P<0.0001$. PND, postnatal day.

(Table 1). However, the number of mitotic cells and the mitosis-karyorrhexis index were increased in the HG group. On the other hand, the number of apoptotic cells (TUNEL assay), the cell width, and the number of cell nuclei were similar between the groups (Table 2).

By now, expression of almost all of the cardiac genes examined was enhanced in the HG group compared with controls (Figure 2). Only the expression of Nppa and Myh3 was similar between the groups. Table 3 shows the changes in gene expression between the day of delivery and at the age of 2 weeks in the HG and control groups.

\section{DISCUSSION}

This experimental study shows that maternal HG during pregnancy modifies expression of the genes that have an important role for cardiac growth, function, and metabolism in the rat offspring. Most of the changes in cardiac gene expression become normal during the first week of life of the offspring. However, by the age of 2 weeks, equivalent to a human infant age of 3-6 months, expression of the genes that regulate cardiac growth, function, and metabolism was again significantly higher in the HG group than in the group of offspring to healthy rats who had had an uneventful pregnancy. These gene expression alterations may have a role in later cardiovascular morbidity.

Maternal high blood glucose during pregnancy correlates with fetal HG in both humans (19) and rats (20) and leads to excess growth of pancreatic $\beta$-cells $(19,20)$. After birth, the glucose supply to the newborns decreases markedly and hyperinsulinemic hypoglycemia ensues in humans $(7,19)$ and rats $(20,21)$. In rats, this lasts only a few hours, after which the newborn becomes normoglycemic (20). In the present study, after birth all rat newborns were fed by euglycemic dams. In rats, pregestational maternal $\mathrm{HG}$ results in fetal cardiac hyperplasia, myocardial remodeling, a transient reduction in cardiac output, and decreased heart rate during the last trimester of pregnancy (9) as well as impaired placental function (22). In the present study, the heart weight of newborns to rats with maternal pregestationally induced HG was higher than that of newborns to control rats. However, the size of the cardiomyocytes cells remained similar. At the same time, cardiac gene expression patterns in the HG group suggest that cardiac development favors slower myosin isotypes and decreased expression of genes involved in the regulation of cardiac contraction, Atp2a2 and Kcnip2, and increased $N p p b$ expression on the day of delivery. Teleologically, exposure to intrauterine $H G$ seems to induce 
Table 3. Neonate cardiac gene expressions and an analysis of differences within the groups on PND0 and 14 (gene/18 S, arbitrary units)

\begin{tabular}{lcccccc}
\hline Variable & \multicolumn{2}{c}{ Control } & & \multicolumn{2}{c}{ Hyperglycemia } \\
\cline { 1 - 2 } \cline { 5 - 6 } \cline { 5 - 6 } Number of pups & 10 & PND0 & PND14 & & PND0 & PND14 \\
\hline Number of dams & 2 & & & & 14 & 13 \\
& & & & 3 & 3
\end{tabular}

Natriuretic peptides

$\begin{array}{lllll}\text { Nppa } & 0.89 \pm 0.46 & 0.77 \pm 0.65 & 1.29 \pm 0.70 & 0.80 \pm 0.86 \\ N p p b & 2.08 \pm 1.31 & 0.66 \pm 0.43^{*} & 3.50 \pm 1.69 & 1.35 \pm 0.66^{* *}\end{array}$

Growth

Tnfrsf12a

$2.82 \pm 1.14 \quad 0.44 \pm 0.23 \dagger 1.79 \pm 0.96 \quad 0.85 \pm 0.28^{*}$

$\begin{array}{ccccc}\text { Myosins } & & & & \\ \text { Myh2 } & 1.28 \pm 0.83 & 0.56 \pm 0.22^{*} & 0.71 \pm 0.50 & 0.95 \pm 0.27 \\ \text { Myh3 } & 1.40 \pm 0.47 & 0.26 \pm 0.17^{\dagger} & 2.07 \pm 1.74 & 0.50 \pm 0.56^{*} \\ \text { Myh6 } & 1.32 \pm 0.35 & 0.89 \pm 0.26^{*} & 0.92 \pm 0.33 & 1.66 \pm 0.51^{* *}\end{array}$

$\begin{array}{ccccc}\text { Contractility } & & & & \\ \text { Kcnip2 } & 0.91 \pm 0.27 & 1.13 \pm 0.41 & 0.49 \pm 0.18 & 2.35 \pm 0.76^{\dagger} \\ \text { Atp2a2 } & 0.99 \pm 0.22 & 1.15 \pm 0.25 & 0.74 \pm 0.19 & 1.83 \pm 0.43^{\dagger}\end{array}$

$\begin{array}{lllll}\text { Metabolism } & & & & \\ \text { Slc2a3 } & 1.63 \pm 0.29 & 0.88 \pm 0.29^{* *} & 0.93 \pm 0.35 & 1.48 \pm 0.18^{\dagger} \\ \text { SIc2a4 } & 0.64 \pm 0.15 & 1.28 \pm 0.38^{* *} & 0.47 \pm 0.27 & 2.47 \pm 0.72^{\dagger} \\ \text { Ucp2 } & 1.24 \pm 0.65 & 1.13 \pm 0.26 & 1.01 \pm 0.35 & 2.12 \pm 0.54^{\dagger} \\ \text { Ucp3 } & 0.26 \pm 0.12 & 2.36 \pm 1.33^{* *} & 0.18 \pm 0.07 & 4.36 \pm 1.53^{\dagger}\end{array}$

Apoptosis

$\begin{array}{lllll}E g l n 3 & 1.06 \pm 0.34 & 0.94 \pm 0.31 & 0.95 \pm 0.32 & 1.87 \pm 0.52^{\dagger}\end{array}$

Atp2a2, sarcoplasmic reticulum Ca2+-ATPase; Egln3, HIF-prolyl hydroxylase 3/egl nine homolog 3; Kcnip2, Kv channel-interacting protein 2; Myh2, adult skeletal heavy chain myosin-2; Myh3, embryonic skeletal heavy chain myosin-3; Myh6, alpha cardiac heavy chain myosin-6; Nppa, A-type/atrial natriuretic peptide gene; Nppb, B-type natriuretic peptide gene; PND, postnatal day; S/c2a3, facilitated glucose transporter 3; S/c2a4, insulin-responsive glucose transporter 4; Tnfrsf12a, tumor necrosis factor receptor superfamily, member 12a; Ucp2, uncoupling protein 2; Ucp3, uncoupling protein 3.

Data are given as means \pm s.d.

${ }^{*} P<0.05 ;{ }^{* *} P<0.001,{ }^{\dagger} P<0.0001$

myocardial contraction that saves energy and oxygen (23). Cardiac expression of genes involved in electrical, contractile, endocrine, and metabolic function is affected by maternal HG near term (9). In the present study, the expression patterns of cardiac genes on the day of delivery were similar to those near term, with the exception for genes involved in cardiac metabolism (Slc2a3, Ucp2, Ucp3), growth (Tnfrsf12a), and contractility $(M y h 2)$. We believe this is due to an increased oxygen partial pressure and altered cardiac loading conditions after birth.
At the age of 1 week, cardiac weight was comparable between the groups, whereas the body weight was lower in the neonatal rats born to dams with pregestational HG. Furthermore, most gene expression changes of the heart detected on the day of delivery had become similar to what was observed in the control group. However, the expression of slower embryonic myosin (Myh3) was reduced in the neonatal rats of HG dams compared with that in control dams. This suggests a transition to faster myosin isoforms in the myocardium.

Two weeks after birth, cardiac and body weights were similar in the offspring of both groups, but the proliferative activity in the myocardium was higher in the maternal HG group. The expression of several cardiac genes was increased, including the genes regulating glucose transporters and uncoupling proteins. At the same time, there is a shift in energy supply from glucose to fatty acids in the cardiomyocytes as a part of the cardiac maturation process (23). Especially Ucp3 seems to be highly expressed when the mitochondrial fatty-acid supply exceeds its oxidative capacity $(24,25)$. In humans, interestingly, excess cardiac growth related to maternal diabetes seems to delay neonatal transition from carbohydrates to fatty acids as a main energy resource in the cardiomyocytes (26). Insulin resistance further increases the supply of fatty acids to the mitochondria (24).

At 2 weeks, the expression of genes involved in cardiac contractility was also increased. A crucial regulator of myocardial excitation and contraction is Atp2a2, the signaling of which requires high concentrations of ATP in its vicinity. Consequently, its expression is significantly reduced in hypoxic conditions (27). In newborn murine hearts, the sarcoendoplasmic ATPase regulation is known to evolve gradually (28). In the present study, the cardiac expression of contractility- and metabolism-involved genes, as well as fast cardiac $\alpha$-myosin (Myh6), increased in the offspring of hyperglycemic dams between 1 and 2 weeks of life, although the expression pattern in the control offspring was opposite. We propose that maternal pregestational HG leads to longterm changes in cardiac gene expression of the offspring.

A few weeks after delivery, the offspring of HG dams are at an increased risk for hypertension, HG, proteinuria, and limited weight gain (24), and, 15 weeks after delivery, the offspring born to dams with HG during pregnancy become insulin-resistant (20), comparable to a state of pre-metabolic type 2 diabetes. In rats, hypertension during the neonatal period causes arterial vasoconstriction later in life (29). In humans, as well, hypertension early in life predisposes to cardiovascular disease in adulthood (30). Our observations showing altered cardiac gene expression patterns at the age of 2 weeks further support the concept that maternal pregestational HG could predispose the offspring to cardiovascular morbidity.

Our experiment has certain limitations. An animal model can only mimic human disease to a certain extent. However, the streptozotocin-induced HG model has been used in experimental studies on diabetes and related conditions for decades, and its limitations and benefits are known $(10,31)$. 
One of the limitations is that this model does not induce all the pathogenic processes of human autoimmune type 1 diabetes-i.e., renal, electrolyte, and lipid homeostasis disturbances (7), oxidative stress (32), and pro-inflammatory processes (33). Furthermore, we do not have data on neonatal blood glucose levels.

In conclusion, our study shows that maternal pregestational HG is associated with altered expression of genes that regulate cardiac growth, function, and metabolism. The gene expression alterationspatterns are mostly normalized by the age of 1 week, only to return to a higher level by 2 weeks of age. Our results suggest that maternal pregestational HG could reprogram the myocardium of the offspring and cause longterm consequences for the cardiac gene expression patterns. This could predispose the offspring to cardiovascular morbidity later in life.

\section{STATEMENT OF FINANCIAL SUPPORT}

This study was supported by grants from the Diabetes Research Foundation of the Finnish Diabetes Association, the Turku University Hospital Research Foundation, the Maud Kuistila Memorial Foundation, the Turku University Foundation, and the National Graduate School of Clinical Investigation.

Disclosure: The authors declare no conflict of interest.

\section{REFERENCES}

1. Thornburg K, Jonker S, O'Tierney $\mathrm{P}$, et al. Regulation of the cardiomyocyte population in the developing heart. Prog Biophys Mol Biol 2011;106:289-99.

2. Li F, Wang X, Capasso JM, Gerdes AM. Rapid transition of cardiac myocytes from hyperplasia to hypertrophy during postnatal development. J Mol Cell Cardiol 1996;28:1737-46.

3. Aman J, Hansson U, Ostlund I, Wall K, Persson B. Increased fat mass and cardiac septal hypertrophy in newborn infants of mothers with wellcontrolled diabetes during pregnancy. Neonatology 2011;100:147-54.

4. Oberhoffer R, Högel J, Stoz F, Kohne E, Lang D. Cardiac and extracardiac complications in infants of diabetic mothers and their relation to parameters of carbohydrate metabolism. Eur J Pediatr 1997;156:262-5.

5. Veille JC, Sivakoff M, Hanson R, Fanaroff AA. Interventricular septal thickness in fetuses of diabetic mothers. Obstet Gynecol 1992;79:51-4.

6. Narchi H, Kulaylat N. Heart disease in infants of diabetic mothers. Images Paediatr Cardiol 2000;2:17-23.

7. Nold JL, Georgieff MK. Infants of diabetic mothers. Pediatr Clin North Am 2004;51:619-37, viii.

8. Corrigan N, Treacy A, Brazil DP, McAuliffe FM. Cardiomyopathy and diastolic dysfunction in the embryo and neonate of a type 1 diabetic mouse model. Reprod Sci 2013;20:781-90.

9. Lehtoranta L, Vuolteenaho O, Laine VJ, et al. Maternal hyperglycemia leads to fetal cardiac hyperplasia and dysfunction in a rat model. Am J Physiol Endocrinol Metab 2013;305:E611-9.

10. Rees DA, Alcolado JC. Animal models of diabetes mellitus. Diabet Med 2005;22:359-70.

11. Burri PH. Structural aspects of postnatal lung development - alveolar formation and growth. Biol Neonate 2006;89:313-22.

12. Mandarim-de-Lacerda CA, Pessanha MG. Stereology of the myocardium in embryos, fetuses and neonates of the rat. Acta Anat 1995;154:261-6.

13. Laine VJ, Nyman KM, Peuravuori HJ, Henriksen K, Parvinen M, Nevalainen TJ. Lipopolysaccharide induced apoptosis of rat pancreatic acinar cells. Gut 1996;38:747-52.
14. Kyto V, Saraste A, Saukko P, et al. Apoptotic cardiomyocyte death in fatal myocarditis. Am J Cardiol 2004;94:746-50.

15. Altunkaynak ME, Altunkaynak BZ, Unal D, Yildirim S, Can I, Unal B. Stereological and histological analysis of the developing rat heart. Anat Histol Embryol 2011;40:402-10.

16. Shimada H, Chatten J, Newton Jr WA, et al. Histopathologic prognostic factors in neuroblastic tumors: definition of subtypes of ganglioneuroblastoma and an age-linked classification of neuroblastomas. J Natl Cancer Inst 1984;73:405-16.

17. Majalahti-Palviainen T, Hirvinen M, Tervonen V, Ilves M, Ruskoaho H, Vuolteenaho O. Gene structure of a new cardiac peptide hormone: a model for heart-specific gene expression. Endocrinology 2000;141: 731-40.

18. Hefti MA, Harder BA, Eppenberger HM, Schaub MC. Signaling pathways in cardiac myocyte hypertrophy. J Mol Cell Cardiol 1997;29:2873-92.

19. Schwartz R, Teramo KA. Effects of diabetic pregnancy on the fetus and newborn. Semin Perinatol 2000;24:120-35.

20. Han J, Xu J, Long YS, Epstein PN, Liu YQ. Rat maternal diabetes impairs pancreatic beta-cell function in the offspring. Am J Physiol Endocrinol Metab 2007;293:E228-36.

21. Nessa A, Rahman SA, Hussain K. Hyperinsulinemic hypoglycemia - the molecular mechanisms. Front Endocrinol 2016;7:29.

22. Lehtoranta L, Vuolteenaho O, Laine J, Polari L, Ekholm E, Räsänen J. Placental structural abnormalities have detrimental hemodynamic consequences in a rat model of maternal hyperglycemia. Placenta 2016;44:54-60.

23. Rajabi M, Kassiotis C, Razeghi P, Taegtmeyer H. Return to the fetal gene program protects the stressed heart: a strong hypothesis. Heart Fail Rev 2007;12:331-43.

24. Cole MA, Murray AJ, Cochlin LE, et al. A high fat diet increases mitochondrial fatty acid oxidation and uncoupling to decrease efficiency in rat heart. Basic Res Cardiol 2011;106:447-57.

25. Chan CB, Harper ME. Uncoupling proteins: role in insulin resistance and insulin insufficiency. Curr Diabetes Rev 2006;2:271-83.

26. Kantor PF, Robertson MA, Coe JY, Lopaschuk GD. Volume overload hypertrophy of the newborn heart slows the maturation of enzymes involved in the regulation of fatty acid metabolism. J Am Coll Cardiol 1999;33:1724-34.

27. Revuelta-López E, Cal R, Herraiz-Martínez A, et al. Hypoxia-driven sarcoplasmic/endoplasmic reticulum calcium ATPase 2 (SERCA2) downregulation depends on low-density lipoprotein receptor-related protein 1 (LRP1)-signalling in cardiomyocytes. J Mol Cell Cardiol 2015;85:25-36.

28. Piquereau J, Novotova M, Fortin D, et al. Postnatal development of mouse heart: formation of energetic microdomains. J Physiol 2010;588: 2443-54.

29. Vessières E, Dib A, Bourreau J, et al. Long lasting microvascular tone alteration in rat offspring exposed in utero to maternal hyperglycaemia. PLoS ONE 2016;11:e0146830.

30. Mitanchez D, Yzydorczyk C, Siddeek B, Boubred F, Benahmed M, Simeoni U. The offspring of the diabetic mother - short- and long-term implications. Best Pract Res Clin Obstet Gynaecol 2015;29: 256-69.

31. Junod A, Lambert AE, Stauffacher W, Renold AE. Diabetogenic action of streptozotocin: relationship of dose to metabolic response. J Clin Invest 1969;48:2129-39.

32. Wentzel P, Ejdesjö A, Eriksson UJ. Maternal diabetes in vivo and high glucose in vitro diminish GAPDH activity in rat embryos. Diabetes 2003;52:1222-8.

33. Sisino G, Bouckenooghe T, Aurientis S, Fontaine P, Storme L, Vambergue A. Diabetes during pregnancy influences Hofbauer cells, a subtype of placental macrophages, to acquire a pro-inflammatory phenotype. Biochim Biophys Acta 2013;1832:1959-68. 